\title{
Do Xpert MTB/RIF Cycle Threshold Values Provide Information about Patient Delays for Tuberculosis Diagnosis?
}

\author{
Willy Ssengooba ${ }^{1,2,3 *}$, Durval Respeito ${ }^{4}$, Edson Mambuque ${ }^{4}$, Silvia Blanco ${ }^{2,4}$, \\ Helder Bulo ${ }^{4}$, Inacio Mandomando $0^{4,5}$, Bouke C. de Jong ${ }^{6}$, Frank G. Cobelens ${ }^{1,7}$, Alberto \\ L. García-Basteiro ${ }^{1,2,4}$
}

1 Department of Global Health and Amsterdam Institute of Global Health and Development, Academic Medical Center, University of Amsterdam, Amsterdam, the Netherlands, 2 ISGlobal, Barcelona Ctr. Int. Health Res. (CRESIB), Hospital Clínic-Universitat de Barcelona, Barcelona, Spain, 3 Department of Medical Microbiology, College of Health Sciences Makerere University, Kampala, Uganda, 4 Centro de Investigação em Saúde de Manhiça, Maputo, Mozambique, 5 Instituto Nacional de Saúde, Ministério da Saúde, Maputo, Mozambique, 6 Mycobacteriology Unit, Institute of Tropical Medicine, Antwerp, Belgium, 7 KNCV Tuberculosis Foundation, The Hague, the Netherlands

* willyssengooba@gmail.com

\section{Abstract}

\section{G openaccess}

Citation: Ssengooba W, Respeito D, Mambuque E, Blanco S, Bulo H, Mandomando I, et al. (2016) Do Xpert MTB/RIF Cycle Threshold Values Provide Information about Patient Delays for Tuberculosis Diagnosis? PLoS ONE 11(9): e0162833. doi:10.1371/ journal.pone.0162833

Editor: Daniela Flavia Hozbor, Universidad Nacional de la Plata, ARGENTINA

Received: June 8, 2016

Accepted: August 29, 2016

Published: September 9, 2016

Copyright: $\odot 2016$ Ssengooba et al. This is an open access article distributed under the terms of the Creative Commons Attribution License, which permits unrestricted use, distribution, and reproduction in any medium, provided the original author and source are credited.

Data Availability Statement: All relevant data are within the paper.

Funding: This work was supported by the Erasmus Mundus Joint Doctorate Program of the European Union through a training grant to WS.

Competing Interests: The authors have declared that no competing interests exist.

\section{Introduction}

Early diagnosis and initiation to appropriate treatment is vital for tuberculosis (TB) control. The XpertMTB/RIF (Xpert) assay offers rapid TB diagnosis and quantitative estimation of bacterial burden through Cycle threshold $(\mathrm{Ct})$ values. We assessed whether the Xpert Ct value is associated with delayed TB diagnosis as a potential monitoring tool for TB control programme performance.

\section{Materials and Methods}

This analysis was nested in a prospective study under the routine TB surveillance procedures of the National TB Control Program in Manhiça district, Maputo province, Mozambique. Presumptive TB patients were tested using smear microscopy and Xpert. We explored the association between Xpert Ct values and self-reported delay of Xpert-positive TB patients as recorded at the time of diagnosis enrolment. Patients with $>60$ days of TB symptoms were considered to have long delays.

\section{Results}

Of 1,483 presumptive TB cases, 580 were diagnosed as TB of whom 505 (87.0\%) were due to pulmonary TB and 302 (94.1\%) were Xpert positive. Ct values (range, 9.7-46.4) showed a multimodal distribution. The median (IQR) delay was 30 (30-45) days. Ct values showed no correlation with delay $\left(R^{2}=0.001, p=0.621\right)$, nor any association with long delays: adjusted odds ratios (AOR) (95\% confidence interval [CI]) comparing to $>28$ cycles 0.99 $(0.50-1.96 ; p=0.987)$ for $23-28$ cycles, $0.93(0.50-1.74 ; p=0.828)$ for $16-22$ cycles; and 
$1.05(0.47-2.36 ; p=0.897)$ for $<16$ cycles. Being HIV-negative (AOR [95\% CI]), 2.05 (1.19$3.51, p=0.009)$ and rural residence $1.74(1.08-2.81, p=0.023)$, were independent predictors of long delays.

\section{Conclusion}

Xpert Ct values were not associated with patient delay for TB diagnosis and cannot be used as an indicator of TB control program performance.

\section{Introduction}

Tuberculosis (TB) remains a global health burden and caused 1.5 million deaths in 2014 globally [1]. Early diagnosis and initiation of effective TB treatment are vital for bringing down TB transmission [2,3]. Delays in diagnosing TB hamper effective TB control, and TB control programmes aim for reducing such delays as much as possible [4]. A readily available measure of diagnostic delay would help TB control programmes in monitoring their performance in this respect.

The Xpert MTB/RIF assay (Xpert), a cartridge-based real-time PCR, offers rapid diagnosis of susceptible TB and resistance-conferring mutations to rifampicin in the $r p o B$ gene within 2 hours of testing. The Xpert technology also offers a quantitative estimation of bacterial burden in form of cycle threshold $(\mathrm{Ct})$ values. Ct values have been found to predict decreasing bacterial burden with increasing immunosuppression and a combination of $\mathrm{Ct}$ values and sputum smear status to be a possible measure of bacterial burden in high HIV-burden settings [5]. Several studies have documented the various factors associated with patient delay for TB diagnosis $[6,7,8,9,10,11,12,13,14]$. Mycobacterial load is likely to increase with patient delay since bacillary burden has been found to grow over time and may have an impact on the novel diagnostic tests $[15,16]$.

Mozambique with a population of 27 million people in 2014, has low case detection rate, below 50\%[17], the estimated TB incidence is around 551 cases per 100,000 population; of these cases, an estimated 58\% are HIV co-infected [18]. By 2014, 24 Xpert machines were operating in the country [1]. A study done in Beira city of Mozambique found the median patient delay for TB diagnosis to be 61 days (28-113)[19].

This study aimed at analyzing whether Ct values are correlated with diagnostic delays and/ or associated with long delays among patients referred for Xpert testing in Mozambique. If such correlation or association were to exist, Ct values could be used as an indicator for monitoring diagnostic delays.

\section{Materials and Methods}

\section{Study setting}

The current study was nested in a prospective surveillance based study embedded in the routine TB patient management procedures of the National TB Control Program for the district of Manhiça, a high TB and HIV burden area in Southern Mozambique [20,21]. The study was conducted by the Manhiça Health Research Center (Centro de Investigação em Saude de Manhiça, CISM) located adjacent to the Manhiça District Hospital, where a majority of the presumptive TB cases from peripheral health centres in the district are referred for diagnosis. 


\section{Study participants}

The study enrolled consecutively presumptive TB cases referred for TB diagnosis at the Manhiça District Hospital from 11 health care centers between August 2013 and August 2014. A presumptive TB case was defined as someone presenting with cough for more than 2 weeks and/or weight loss and/or fever and/or night sweats for more than two weeks, regardless of having been previously treated for TB. Although Xpert was not routinely used in the country at that time, it was used in this research context, together with AFB smear on Ziehl Neelsen microscopy, as an initial test for any specimen (mostly sputa) provided by a presumptive TB case. Culture was only done for diagnosis in TB previously treated patients (former WHO Category 2 patients) or new TB cases who were AFB smear positive at month 5 of follow up. AFB smears were processed in the Biosafety Level III TB laboratory at CISM. On a ratio of 1:2 $\mathrm{mL}$ of sample to sample reagents, incubated at room temperature for 15 minutes, $1 \mathrm{~mL}$ of the mixture was added to the Xpert cartridge and analyzed in Xpert system according to manufacturer's guide. Xpert Ct values were taken as the mean of PCR cycles obtained from the five probes (A-E) of the Xpert machines. Ct values inversely correlate with bacterial load i.e. lower Ct values represent a higher starting concentration of DNA template whereas higher Ct values represent a lower concentration of DNA template. The mean Ct values are also categorized by the Xpert system semi-quantitatively in relation to sample positivity as very low ( $>28$ cycles), low (23-28 cycles), medium (16-22 cycles) and high ( $<16$ cycles)[22].

Study specific questionnaires were implemented to capture the most important variables. Demographic and clinical data were collected at enrolment.

\section{Operational definitions}

Patient delay for TB diagnosis was defined as reported total duration of symptoms at the time of study enrolment. By the time of enrolment the patient could have had several diagnostic visits to the clinic where the current TB diagnosis was made, as well as sought care from several health services providers. For these reasons as well as the wide variation in literature about patient delay for TB diagnosis $[6,7,8,9,10,11,12,13,14]$, we operationalized long delay to be $\geq 60$ days with TB symptoms before TB diagnosis before TB diagnosis before current study data analysis. This was considered as delay long enough to significantly impact on the TB control programmes.

We considered cough as the main symptom and if absent, duration of the other TB symptom(s) was considered. For the current analysis, a TB case was defined as a study participant positive with Xpert test.

\section{Data management and analysis}

The data collected from presumptive TB cases and TB cases were kept in paper forms (CRFs), which were checked by the study coordinators and study nurse at the end of each week for quality assurance. Completed forms were double computer-entered at CISM. Data were exported to Stata, (Stata Corp LP, College Station TX, USA) for analysis of frequencies and proportions of participants' characteristics and median symptom duration were compared. We used the Wilcoxon ranksum test and the Kruskall-Wallis test to compare delays between groups, and Spearman's correlation coefficient to express the correlation between delays and $\mathrm{Ct}$ values. In the analysis of long delays we adjusted the association between categorized Ct values and delays of $\geq 60$ days duration for confounding variables in a multivariate logistic regression model. In a secondary analysis we explored independent associations with long delays by including in a similar model all covariates with a $\mathrm{p}$-value $<0.2$ at univariate analysis, using 
back-ward elimination to arrive at a final model that contained variables with a p-value $<0.05$. Reported model p-values were based on the likelihood ratio test.

\section{Ethical consideration}

The study was approved by the CISM's Internal Scientific Committee, CISM's Institutional Bioethics Committee for Health (CIBS-Comité Institucional de Bioética para a Saúde) and the National Bioethics Committee for Health (CNB-Comité Nacional de Bioética para a Saúde) of Mozambique. All individuals gave a written informed consent before participation in the study.

\section{Results}

\section{Characteristics of participants}

A total of 1483 presumptive TB cases were enrolled in the current study of whom 580 were diagnosed as TB. 505(87.0\%) were due to pulmonary TB, 204 (40.4\%) of whom were smear positive and 321 (63.6\%) were positive for Xpert, Fig 1.

Of the 321 Xpert positive TB patients recorded, data for Ct value were available for $302 \mathrm{~TB}$ patients of whom 176 (58\%) were male, median age was 35 (interquartile range 33-37) years, 154 (51\%) were from urban areas of the district 222 (74\%) were HIV-positive and 14 (4.6\%) had $r p o B$ mutation. The patient characteristics and median delay are shown in Table 1.

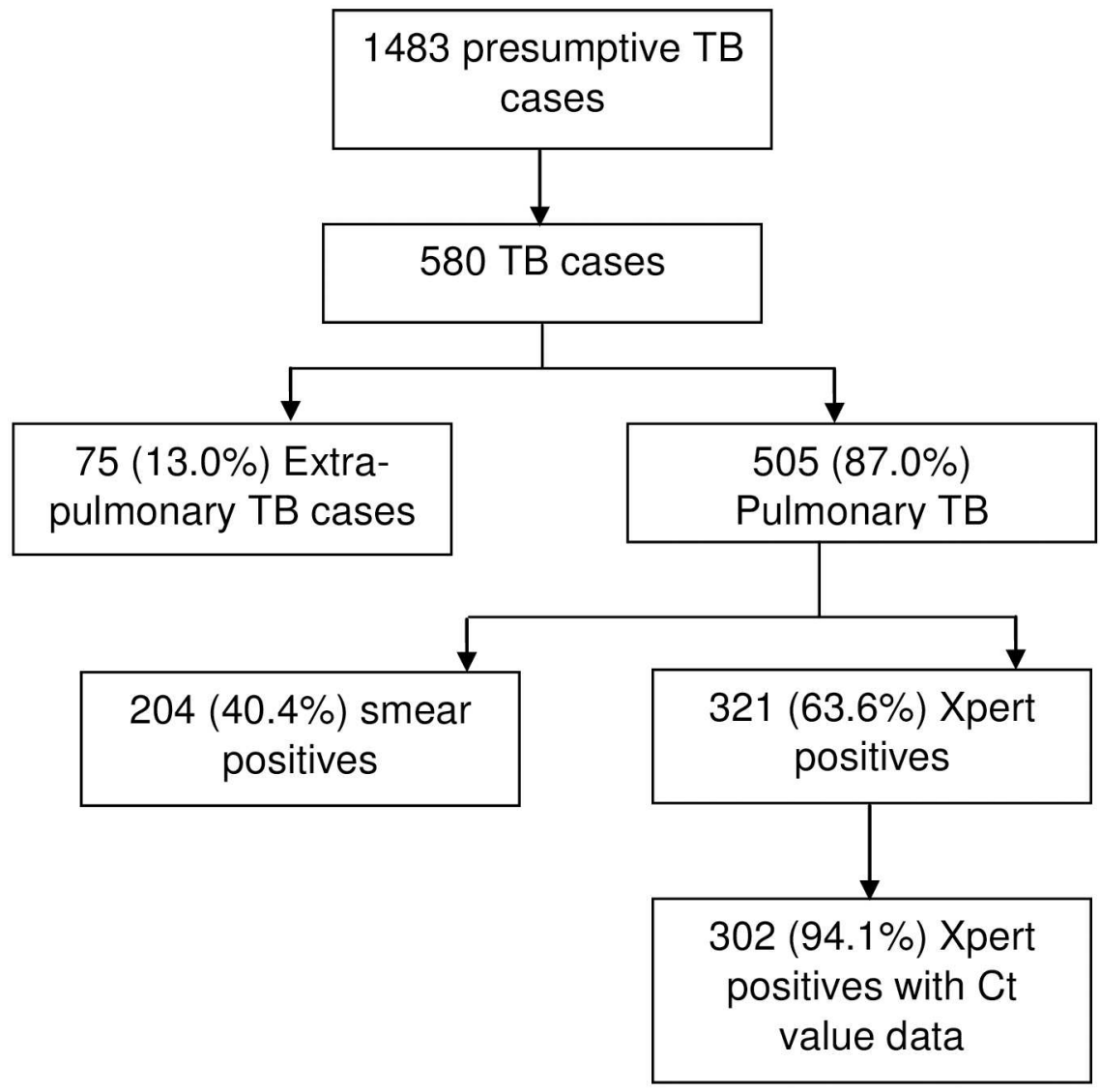

Fig 1. Flow diagram showing XpertMTB/RIF test positive tuberculosis participants. $T B=$ tuberculosis, $\mathrm{EPTB}=$ extra pulmonary tuberculosis, $\mathrm{PTB}=$ pulmonary tuberculosis.

doi:10.1371/journal.pone.0162833.g001 
Table 1. Demographic and clinical-demographic characteristics and diagnostic delay of pulmonary TB patients.

\begin{tabular}{|c|c|c|c|}
\hline Characteristic & Frequency, n (\%) & Diagnostic delay Median (IQR) & $P$ value \\
\hline \multicolumn{4}{|l|}{ Sex } \\
\hline Female & $126(41.7)$ & $30(30-60)$ & \\
\hline Male & $176(58.3)$ & $30(30-60)$ & 0.555 \\
\hline \multicolumn{4}{|l|}{ Age category } \\
\hline$\leq 35$ & $146(48.5)$ & $30(30-60)$ & \\
\hline$>35$ & $155(51.5)$ & $30(30-45)$ & 0.934 \\
\hline Median age (IQR) & $35(33-37)$ & & \\
\hline \multicolumn{4}{|l|}{ Residence } \\
\hline Urban & $154(51.0)$ & $30(30-30)$ & \\
\hline Rural & $148(49.0)$ & $60(30-60)$ & 0.041 \\
\hline \multicolumn{4}{|l|}{ History of TB treatment } \\
\hline New & $254(84.1)$ & $30(30-45)$ & \\
\hline Previously treated & $48(15.9)$ & $45(30-60)$ & 0.177 \\
\hline \multicolumn{4}{|l|}{ Presence of cough } \\
\hline Yes & $290(96.0)$ & $30(7-131)$ & \\
\hline No & $12(4.0)$ & $30(30-53)$ & 0.834 \\
\hline \multicolumn{4}{|l|}{ Fever } \\
\hline Yes & $248(82.1)$ & $30(30-60)$ & 0.854 \\
\hline No & $54(17.9)$ & $30(30-60)$ & \\
\hline \multicolumn{4}{|l|}{ Night sweats } \\
\hline Yes & $246(81.7)$ & $30(30-60)$ & \\
\hline No & $55(18.3)$ & $30(30-60)$ & 0.790 \\
\hline \multicolumn{4}{|l|}{ HIV-status } \\
\hline Positive & $222(74.5)$ & $30(30-60)$ & \\
\hline Negative & $76(25.5)$ & $60(30-60)$ & 0.093 \\
\hline \multicolumn{4}{|l|}{ ON ART } \\
\hline Yes & $80(36.0)$ & $30(30-60)$ & 0.160 \\
\hline No & $142(64.0)$ & $30(30-30)$ & \\
\hline \multicolumn{4}{|l|}{ CD4 cell count } \\
\hline$>200$ cells $/ \mathrm{mm}^{3}$ & $84(44.9)$ & $30(30-45)$ & \\
\hline $100-200$ cells $/ \mathrm{mm}^{3}$ & $35(18.7)$ & $30(30-60)$ & 0.708 \\
\hline$<100$ cells $/ \mathrm{mm}^{3}$ & $68(36.4)$ & $30(30-41)$ & 0.729 \\
\hline \multicolumn{4}{|l|}{ Taking cotrimoxazole } \\
\hline Yes & $28(14.1)$ & $30(21-60)$ & \\
\hline No & $170(85.9)$ & $30(30-30)$ & 0.825 \\
\hline \multicolumn{4}{|l|}{ Smear results } \\
\hline Negative & $119(39.7)$ & $30(30-30)$ & 0.086 \\
\hline Positive & $181(60.3)$ & $30(30-60)$ & \\
\hline \multicolumn{4}{|l|}{ XpertMTB/RIF Ct value } \\
\hline Very low (>28 cycles) & $73(24.2)$ & $30(30-60)$ & \\
\hline Low (23-28 cycles) & $75(24.8)$ & $30(30-60)$ & 0.562 \\
\hline Medium ( $16-22$ cycles) & $114(37.7)$ & $30(30-60)$ & 0.991 \\
\hline High (<16 cycles) & $40(13.3)$ & $30(30-60)$ & 0.951 \\
\hline
\end{tabular}

$\mathrm{TB}=$ tuberculosis, IQR = Interquartile range $. \mathrm{Ct}=$ cycle threshold doi:10.1371/journal.pone.0162833.t001 
Fig2a

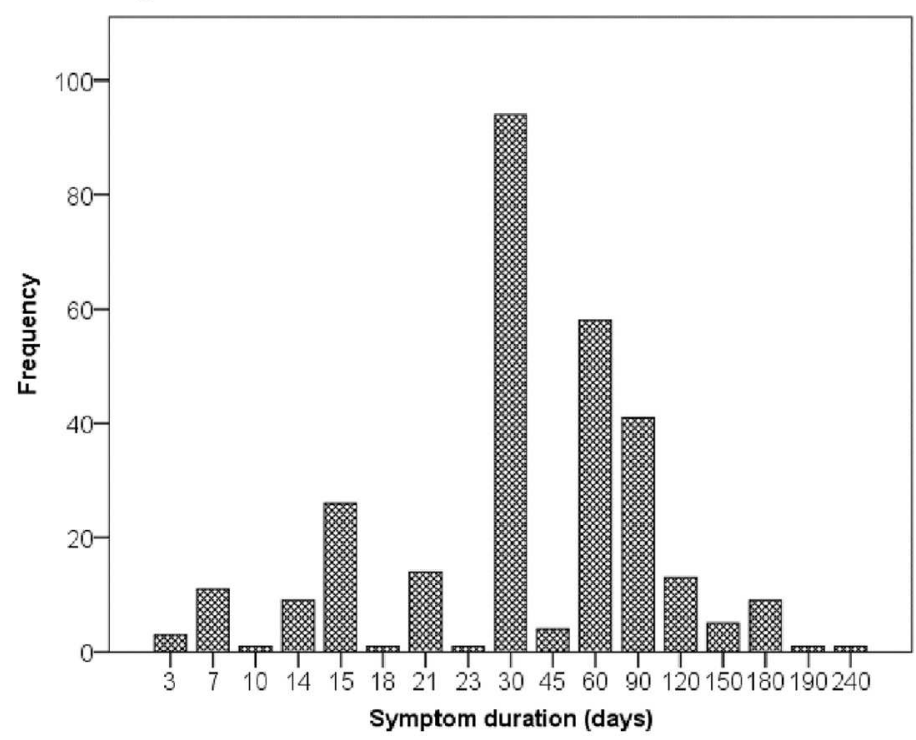

Fig2b

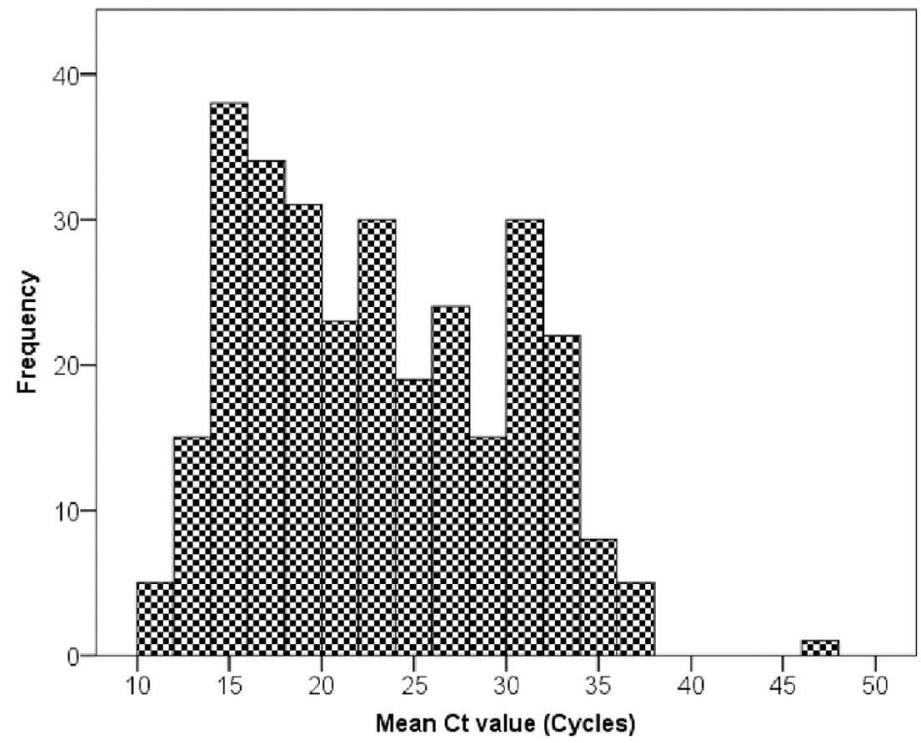

Fig 2. 2a: Distribution of the reported symptom duration, Fig2b: Distribution of the Xpert mean Ct values.

\section{Distribution of patient delay and $\mathrm{Ct}$ values}

Symptom duration showed a skewed distribution with a median of 30 days and an interquartile range of 30 to 45 days,Fig 2a; 37\% had medium (16-22 cycles) Xpert Ct values. Ct values showed a multimodal distribution with peaks around values of 15, 25 and 33 cycles, Fig 2b. There was no correlation between Ct values and delays (Spearman $\mathrm{R}^{2}=0.001, \mathrm{p}=0.612$, Fig 3 ). Log-transforming the delays did not improve the correlation $\left(\mathrm{R}^{2}<0.001, \mathrm{p}=0.798\right)$.

\section{Association of long diagnostic delay with $\mathrm{Ct}$ values}

In univariate analysis, $\mathrm{Xpert} C t$ values showed no significant association with long delays, with adjusted odds ratios (AOR) (95\% confidence interval [CI]) comparing to $>28$ cycles 0.99 $(0.50-1.96 ; \mathrm{p}=0.987)$ for $23-28$ cycles, $0.93(0.50-1.74 ; \mathrm{p}=0.828)$ for $16-22$ cycles; and 1.05 $(0.47-2.36 ; \mathrm{p}=0.897)$ for $<16$ cycles. After multivariate adjustment for confounding by residence and HIV status the association remained absent, Table 2.

\section{Predictors of long diagnostic delay}

Delays (median, IQR) differed significantly between patients residing in rural parts of the district $(60,30-60)$ and patients residing in urban parts $(30,30-30 ; p=0.041)$. There were no significant differences among other demographic and patient characteristics (Table 1).

Factors associated with long patient delay in the multivariate analysis also included being HIV-negative (AOR [95\% CI]), $2.05(1.19-3.51, \mathrm{p}=0.009)$ and residence in rural area of Manhiça district $1.74(1.08-2.81, \mathrm{p}=0.023)$, Table 2.

\section{Discussion}

We found that among pulmonary TB patients in Manhiça district of Mozambique referred for Xpert testing, Xpert Ct values were not associated with the reported duration of TB symptom duration, nor did they predict long delays for TB diagnosis of $>60$ days. Long delays were independently associated with rural residence and HIV-negative status. 


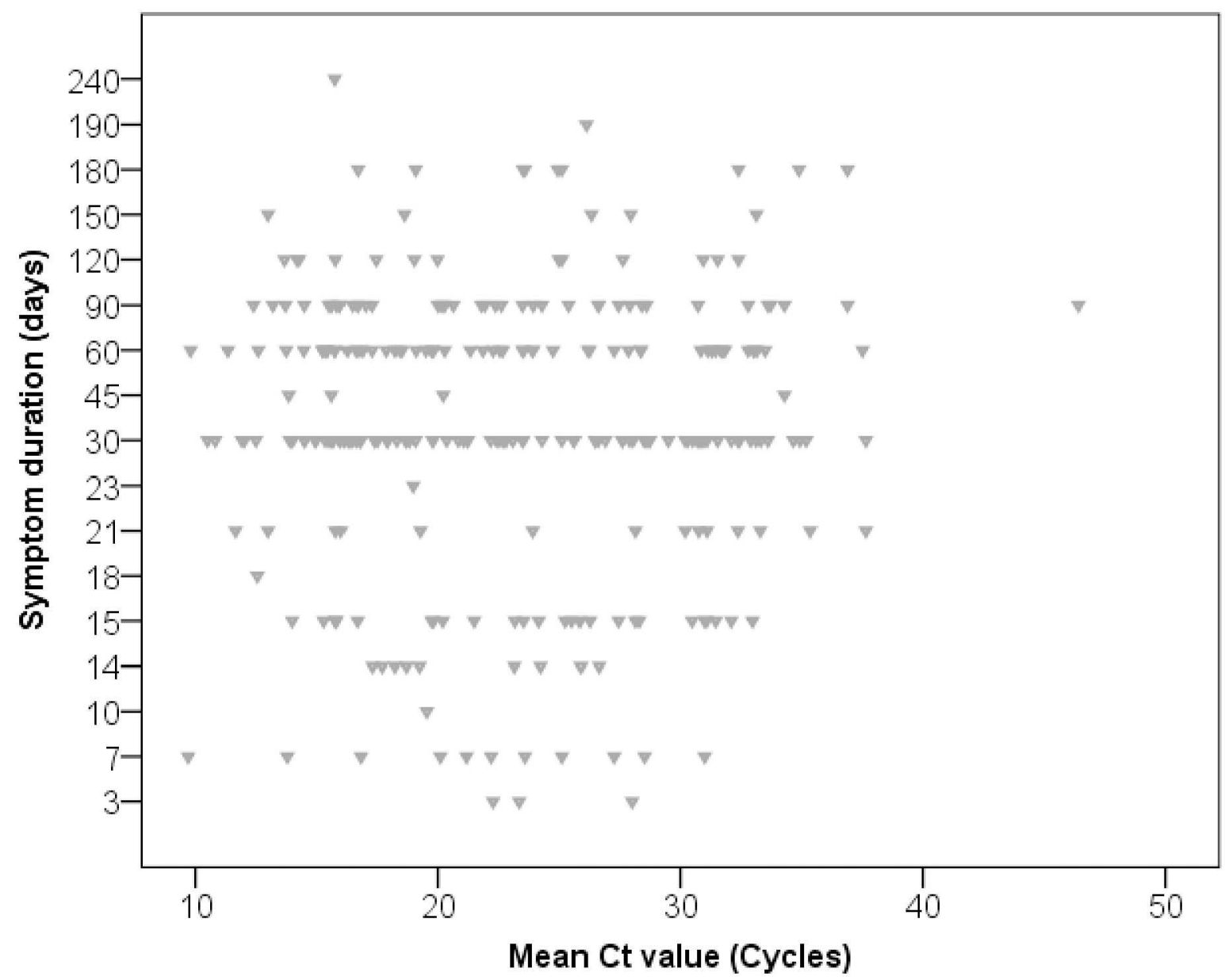

Fig 3. Scatter plot of individuals' mean CT value among those testing XpertMTB/RIF positive by reported symptom duration (Spearman $R^{2}=0.001, p=0.612$ ).

doi:10.1371/journal.pone.0162833.g003

Few studies have looked at additional information that can be generated from routine use of the Xpert assay to inform TB control programs. Previous study found Xpert Ct values to correlate with bacillary burden in the lungs, i.e. increasing bacillary load with decreasing Xpert $\mathrm{Ct}$ values [5]. Measures to estimate patient delay independently from what is reported by the patient would be useful as a tool for monitoring TB control program performance with regard to reducing diagnostic delays. Clearly, Xpert Ct values cannot be used for this purpose.

Table 2. Multivariate predictors of patient delay for TB diagnosis in Manhiça district, Maputo Mozambique.

\begin{tabular}{l|c|c|l}
\hline Variables & Odds ratio & P value & {$[95 \%$ Cl] } \\
\hline Ct 23-28 Cycles & 0.99 & 0.987 & $0.504-1.962$ \\
\hline Ct 16-22 Cycles & 0.93 & 0.828 & $0.499-1.745$ \\
\hline Ct $<16$ Cycles & 1.05 & 0.897 & $0.471-2.362$ \\
\hline HIV-negative & 2.05 & 0.009 & $1.193-3.512$ \\
\hline Rural residence & 1.74 & 0.023 & $1.078-2.811$ \\
\hline
\end{tabular}

$\mathrm{Ct}=$ cycle threshold, $\mathrm{Cl}=$ Confidence interval

doi:10.1371/journal.pone.0162833.t002 
The lack of observed association between Ct values and diagnostic delays may imply that longer delays do not lead to higher bacterial burden, at least not at individual patient level, and that it is the individual clinical trajectory rather than duration of disease that predicts the bacterial burden at diagnosis $[23,24]$. Alternatively, or in addition, it may imply that the reported duration of symptoms does not reflect the duration of disease. This may be due to poor recall by patients and/or relatively long and variable periods of subclinical disease before symptoms arise[25], although subclinical disease is more likely to be missed in routine than in providerinitiated symptom screening for tuberculosis [26]. We investigated other predictors of patient delay for TB diagnosis and found rural residence and being HIV-negative to predict patient delay for TB diagnosis. It is worth noting that Xpert testing in Manhiça is only available in the district town, so rural patients first need to be referred and then take time to travel to town for the Xpert test. This was in agreement with a previous study that found rural residence as a risk factor of patient delay for TB diagnosis [13,27]. Previous studies documented HIV-positive individuals to have less patient delay compared to HIV-negative [12,28]. Previous studies have noted that HIV-positive individuals are more likely to suffer serious TB symptoms which prompt them to seek care earlier than HIV-negative individuals [26,28]. An alternative hypothesis is that TB patients who are HIV-positive and wait too long to be diagnosed die at home. Since there is capacity for smear microscopy in peripheral health centers in Manhiça district, replacing AFB with centralized Xpert may increase TB diagnostic delays [29].

Our study has some limitations. First, the delay as reported by patients was retrospectively analysed from the collected data. The reported duration may have suffered from recall bias for delays and "digit preference" (e.g. 2 weeks, 2 months) in delay ascertainment. Secondly, the findings may not represent delays among presumptive TB patients in Mozambique, but rather a selection of patients in the district referred for Xpert reflecting the local diagnostic and referral algorithm. This limits the conclusions drawn from our secondary analysis but not from our primary analysis, since the latter aimed to look at a biological phenomenon that is expected to exist independent of representativeness of the patients included in the current study. We did not collect data concerning the intervals of health care contacts to the current study yet patients may have contacted other health care facilities and received antibiotics, which may have resolved partly some TB symptoms before the current visit. Finally, we took the mean CT values from the five probes of Xpert Ct for predicting patient delay and had low prevalence 14/ $302(4.6 \%)$ of $r p o B$ mutation, however, in settings with high rifampicin resistance, these may be analysed separately or participants eliminated from the analysis as rifampicin resistance may alter the results.

In conclusion, in our study Xpert Ct values did not predict patient delay for TB diagnosis, and are not useful for monitoring $\mathrm{TB}$ control program performance with regard to reducing diagnostic delay.

\section{Acknowledgments}

This work was supported by the Erasmus Mundus Joint Doctorate Program of the European Union through a training grant to WS. We thank all study participants in the district of Manhiça for their participation in the study and the National Control Program (Dr Ivan Manhiça, Luis Faife) and Health Services of the district (Fausta Temba) for their support. The authors wish to thank the CISM laboratory \& health care staff, as well as data clerks involved in the study

\section{Author Contributions}

Conceptualization: WS FGC ALGB IM BdJ. 
Data curation: WS ALGB DR IM.

Formal analysis: WS FGC ALGB IM BdJ.

Funding acquisition: WS FGC ALGB IM BdJ.

Investigation: WS ALGB DR IM.

Methodology: WS FGC ALGB IM BdJ.

Project administration: WS ALGB DR IM.

Resources: WS ALGB DR IM FGC.

Software: WS FGC ALGB.

Supervision: WS FGC ALGB IM BdJ.

Validation: WS FGC ALGB.

Visualization: WS FGC ALGB IM BdJ.

Writing - original draft: WS.

Writing - review \& editing: WS FGC ALGB IM DR HB SB EM.

\section{References}

1. World Health Organization. Global tuberculosis report2014. http://apps.who.int/iris/bitstream/10665/ 137094/1/9789241564809_eng.pdf Accessed September 26-2015.

2. Riley RL, Mills CC, Nyka W, Weinstock N, Storey PB, Sultan LU, et al. (1995) Aerial dissemination of pulmonary tuberculosis. A two-year study of contagion in a tuberculosis ward. 1959. Am J Epidemiol 142: 3-14. PMID: 7785671

3. Dharmadhikari AS, Mphahlele M, Venter K, Stoltz A, Mathebula R, Masotla T, et al. (2014) Rapid impact of effective treatment on transmission of multidrug-resistant tuberculosis. Int J Tuberc Lung Dis 18: 1019-1025. doi: 10.5588/ijtld.13.0834 PMID: 25189547

4. Yuen CM, Amanullah F, Dharmadhikari A, Nardell EA, Seddon JA, Vasilyeva I, et al. (2015) Turning off the tap: stopping tuberculosis transmission through active case-finding and prompt effective treatment. Lancet 386: 2334-2343. doi: 10.1016/S0140-6736(15)00322-0 PMID: 26515675

5. Hanrahan CF, Theron G, Bassett J, Dheda K, Scott L, Stevens W, et al. (2014) Xpert MTB/RIF as a measure of sputum bacillary burden. Variation by HIV status and immunosuppression. Am J Respir Crit Care Med 189: 1426-1434. doi: 10.1164/rccm.201312-21400C PMID: 24786895

6. Sendagire I, Schim Van der Loeff M, Mubiru M, Konde-Lule J, Cobelens F (2010) Long delays and missed opportunities in diagnosing smear-positive pulmonary tuberculosis in Kampala, Uganda: a cross-sectional study. PLoS One 5: e14459. doi: 10.1371/journal.pone.0014459 PMID: 21206746

7. Buregyeya E, Criel B, Nuwaha F, Colebunders R (2014) Delays in diagnosis and treatment of pulmonary tuberculosis in Wakiso and Mukono districts, Uganda. BMC Public Health 14: 586. doi: 10.1186/ 1471-2458-14-586 PMID: 24916459

8. Makwakwa L, Sheu ML, Chiang CY, Lin SL, Chang PW (2014) Patient and health system delays in the diagnosis and treatment of new and retreatment pulmonary tuberculosis cases in Malawi. BMC Infect Dis 14: 132. doi: 10.1186/1471-2334-14-132 PMID: 24606967

9. Gosoniu GD, Ganapathy S, Kemp J, Auer C, Somma D, Karim F, et al. (2008) Gender and socio-cultural determinants of delay to diagnosis of TB in Bangladesh, India and Malawi. Int J Tuberc Lung Dis 12: 848-855. PMID: 18544215

10. Belkina TV, Khojiev DS, Tillyashaykhov MN, Tigay ZN, Kudenov MU, Tebbens JD, et al. (2014) Delay in the diagnosis and treatment of pulmonary tuberculosis in Uzbekistan: a cross-sectional study. BMC Infect Dis 14: 624. doi: 10.1186/s12879-014-0624-y PMID: 25421106

11. Lin X, Chongsuvivatwong V, Geater A, Lijuan R (2008) The effect of geographical distance on TB patient delays in a mountainous province of China. Int J Tuberc Lung Dis 12: 288-293. PMID: 18284834 
12. Osei $E$, Akweongo $P$, Binka $F$ (2015) Factors associated with DELAY in diagnosis among tuberculosis patients in Hohoe Municipality, Ghana. BMC Public Health 15: 721. doi: 10.1186/s12889-015-1922-z PMID: 26220804

13. Mahendradhata $Y$, Syahrizal BM, Utarini $A$ (2008) Delayed treatment of tuberculosis patients in rural areas of Yogyakarta province, Indonesia. BMC Public Health 8: 393. doi: 10.1186/1471-2458-8-393 PMID: 19036164

14. Takarinda KC, Harries AD, Nyathi B, Ngwenya M, Mutasa-Apollo T, Sandy C, (2015) Tuberculosis treatment delays and associated factors within the Zimbabwe national tuberculosis programme. BMC Public Health 15: 29. doi: 10.1186/s12889-015-1437-7 PMID: 25631667

15. Lawn SD, Kerkhoff AD, Wood R Progression of subclinical culture-positive tuberculosis to symptomatic disease in HIV-infected individuals. AIDS 25: 2190-2191.

16. Arinaminpathy N, Dowdy D (2015) Understanding the incremental value of novel diagnostic tests for tuberculosis. Nature 528: S60-67. doi: 10.1038/nature16045 PMID: 26633767

17. Lopez-Varela E, Augusto OJ, Guerra L, Respeito D, Sacoor C, Sacarlal J, et al. Low paediatric tuberculosis case detection rate in Southern Mozambique. Eur Respir J 47: 1003-1005. doi: 10.1183/ 13993003.01454-2015 PMID: 26699721

18. Garcia-Basteiro AL, Lopez-Varela E, Manhica I, Macete E, Alonso PL (2014) Mozambique faces challenges in the fight against tuberculosis. Lancet 383: 215-216.

19. Saifodine A, Gudo PS, Sidat M, Black J (2014) Patient and health system delay among patients with pulmonary tuberculosis in Beira city, Mozambique. BMC Public Health 13: 559.

20. Garcia-Basteiro AL, Lopez-Varela E, Respeito D, Gonzalez R, Naniche D, Manhica I, et al. (2015) High tuberculosis burden among people living with HIV in southern Mozambique. Eur Respir J 45: 547-549. doi: 10.1183/09031936.00145714 PMID: 25395030

21. Gonzalez R, Munguambe K, Aponte J, Bavo C, Nhalungo D, Macete E, et al. High HIV prevalence in a southern semi-rural area of Mozambique: a community-based survey. HIV Med 13: 581-588. doi: 10. 1111/j.1468-1293.2012.01018.x PMID: 22500780

22. Xpert ${ }^{\mathbb{R}}$ MTB/RIF-Evidence-Based tuberculosis Diagnosis. Xpert ${ }^{\mathrm{R}}$ MTB/RIF; Two-hour detection of MTB and resistance to rifampicin. Available at: http://tbevidence.org/documents/rescentre/sop/ XpertMTB_Broch_R9_EU.pdf; accessed 5th April 2016.

23. Marino S, Gideon HP, Gong C, Mankad S, McCrone JT, Lin P L, et al. Computational and Empirical Studies Predict Mycobacterium tuberculosis-Specific T Cells as a Biomarker for Infection Outcome. PLoS Comput Biol 12: e1004804. doi: 10.1371/journal.pcbi.1004804 PMID: 27065304

24. Tiemersma EW, van der Werf MJ, Borgdorff MW, Williams BG, Nagelkerke NJ (2011) Natural history of tuberculosis: duration and fatality of untreated pulmonary tuberculosis in HIV negative patients: a systematic review. PLoS One 6: e17601. doi: 10.1371/journal.pone.0017601 PMID: 21483732

25. Esmail H, Barry CE 3rd, Young DB, Wilkinson RJ The ongoing challenge of latent tuberculosis. Philos Trans R Soc Lond B Biol Sci 369: 20130437. doi: 10.1098/rstb.2013.0437 PMID: 24821923

26. Corbett EL, Zezai A, Cheung YB, Bandason T, Dauya E, Raviglione MC, et al. (2010) Provider-initiated symptom screening for tuberculosis in Zimbabwe: diagnostic value and the effect of HIV status. Bull World Health Organ 88: 13-21.

27. Sreeramareddy CT, Qin ZZ, Satyanarayana S, Subbaraman R, Pai M (2014) Delays in diagnosis and treatment of pulmonary tuberculosis in India: a systematic review. Int J Tuberc Lung Dis 18: 255-266. doi: 10.5588/ijtld. 13.0585 PMID: 24670558

28. Ngamvithayapong J, Yanai H, Winkvist A, Diwan V (2001) Health seeking behaviour and diagnosis for pulmonary tuberculosis in an HIV-epidemic mountainous area of Thailand. Int J Tuberc Lung Dis 5: 1013-1020. PMID: 11716337

29. Cohen GM, Drain PK, Noubary F, Cloete C, Bassett IV (2014) Diagnostic delays and clinical decision making with centralized Xpert MTB/RIF testing in Durban, South Africa. J Acquir Immune Defic Syndr 67: e88-93. 